\title{
Síndrome de Cushing exógeno e insuficiencia adrenal relacionada con consumo de producto natural
}

\author{
Exogenous Cushing's syndrome and adrenal \\ insufficiency related to consumption of alternative \\ medicinal product
}

\author{
Mónica Zuluaga-Quintero, Alex Ramírez, Andrés Palacio, \\ José Fernando Botero, Absalón Clavijo • Medellín (Colombia)
}

\begin{abstract}
Resumen
La insuficiencia adrenal es una endocrinopatía cuya principal causa se debe a la deficiencia en la producción de hormona adrenocorticotrópica, generalmente explicada por factores iatrogénicos y en menor número de casos al defecto primario de la glándula adrenal (enfermedad de Adisson). Su diagnóstico y la búsqueda de una etiología específica es muy importante, dado las implicaciones a largo plazo que conlleva por necesidad de suplencia hormonal, manejo en condiciones críticas, riesgo de muerte y compromiso de la actividad laboral. Presentamos el caso de una mujer quien consultó por ganancia excesiva de peso, signos de hipercortisolismo e hipertensión, se documentó una probable fuente exógena de esteroides, encontrando un cortisol sérico suprimido. Dos años antes estaba recibiendo artrivid PLUS, un producto natural para el manejo del dolor articular. Después de suspender ésta medicación presentó síntomas compatibles con insuficiencia adrenal, requiriendo el reinicio de prednisolona, con mejoría clínica y logrando su desmonte gradual posterior. (Acta Med Colomb 2017; 42: 243-246)
\end{abstract}

Palabras clave: glándulas suprarrenales, hidrocortisona, síndrome de Cushing

\begin{abstract}
Adrenal insufficiency is an endocrinopathy whose main cause is due to the deficiency in the production of adrenocorticotropic hormone, usually explained by iatrogenic factors and in a smaller number of cases to the primary defect of the adrenal gland (Adisson's disease). Diagnosis and the search for a specific etiology is very important, given the long-term implications involved by the need of hormone replacement, management in critical conditions, risk of death and the compromise of work activity. The case of a woman who consulted for excessive weight gain, signs of hypercortisolism and hypertension, is presented. A probable exogenous source of steroids was documented, finding a suppressed serum cortisol. Two years before she was receiving artrivid PLUS, a natural product for joint pain management. After stopping this medication, she presented symptoms compatible with adrenal insufficiency, requiring the resumption of prednisolone, with clinical improvement and achieving its subsequent gradual removal. (Acta Med Colomb 2017; 42: 243-246)

Keywords: adrenal glands, hydrocortisone, Cushing's syndrome
\end{abstract}

Dra. Mónica Zuluaga-Quintero: Medicina Interna, Hospital Pablo Tobón Uribe, Universidad Pontificia Bolivariana; Dres. Alex Ramírez Rincón, Andrés Palacio y José Fernando Botero: Endocrinólogos, Clínica Integral de Diabetes, Grupo de Investigación IDEAS, Universidad Pontificia Bolivariana; Dr. Absalón Clavijo: Residente Medicina Interna. Universidad Pontificia Bolivariana. Medellín (Colombia).

Correspondencia. Dra. Mónica Zuluga-Quintero. Medellín (Colombia).

E-mail: mzulu28@hotmail.com

Recibido: 12/III/2017 Aceptado: 3/XI/2017

\section{Introducción}

El síndrome de Cushing (SC) es causado por un exceso en los niveles circulantes de cortisol que puede ser secundario al consumo de hidrocortisona, prednisona, dexametasona u otros glucocorticoides sintéticos por largos periodos de tiempo. Situación frecuente en la práctica clínica, debido a diferentes condiciones médicas, inflamatorias, neoplásicas, donde tienen un efecto terapéutico óptimo pero también un importante perfil de efectos adversos, ocasionando cambios en la apariencia facial, incremento de la grasa abdominal y en dorso, debilidad muscular proximal, estrías violáceas, acné, hirsutismo, baja densidad mineral ósea, fracturas, oligomenorrea, depresión, ansiedad, ganancia de peso, infección, eventos embólicos, resistencia a la insulina, 
diabetes, dislipidemia e hipertensión (1). La insuficiencia adrenal (IA) es menos reconocida, presentándose con síntomas generales como fatiga, náuseas, anorexia, en algunos casos hiperpigmentación, vitiligo, hipoglicemia, hiponatremia, hipercalemia, pérdida de peso e hipotensión, incluyendo una manifestación grave con crisis adrenal que podría conllevar a un desenlace fatal $(2,3)$.

La supresión del eje hipotálamo - hipófisis - adrenal puede ocurrir con dosis fisiológicas o mayores de esteroides, que inhiben la producción endógena de hormona liberadora de corticotropina (CRH) y hormona adrenocorticotrópica $(\mathrm{ACTH})$, resultando en hipoplasia adrenal o atrofia con un tiempo indeterminado para recuperar la función del eje y tamaño de la glándula, el cual varía entre días hasta un año o más, independiente de la dosis o tiempo de uso del esteroide $(3,4)$. En una revisión sistemática de la literatura que incluyó 3166 pacientes quienes recibieron esteroides, la prevalencia de IA fue entre 14 y $63 \%$ (media 37\%), reportada hasta tres años después de la suspensión del esteroide; sin embargo, existe heterogeneidad entre estudios respecto al tipo de corticoide, dosis y método diagnóstico utilizado para poder extraer una conclusión universal (5). Un cortisol sérico en la mañana entre las seis y ocho horas mayor de $18 \mathrm{ug} / \mathrm{mL}$ descarta IA, si es menor de $5 \mathrm{ug} / \mathrm{dL}$ es diagnóstico y la determinación de ACTH ayuda a distinguir entre causa primaria o secundaria al estar sus valores aumentados $(>100 \mathrm{pg} / \mathrm{mL})$ o disminuidos $(<20 \mathrm{pg} / \mathrm{mL})$ respectivamente (2); pero en caso de un valor de cortisol en rango indeterminado, éste se debe confirmar con alguno de los cuatro estudios aprobados: prueba de tolerancia a la insulina, uso de metirapona en la noche (no disponible en Colombia), estudio con $\mathrm{CRH}$, estos tres evalúan la función del eje, siendo más común el uso de estímulo con 250 ug de ACTH sintética, el cual correlaciona bien con el estudio de tolerancia a insulina $(2,6,7)$. La hidrocortisona es el corticoide de reemplazo preferido por mejor perfil de seguridad y su efecto mineralocorticoide, 15-25 mg/ día divido en tres dosis repartidas 30 minutos antes de levantarse, a las 12:00 a 14:00 horas y a las 18:00 horas, es el régimen más fisiológico; No obstante, en Colombia no se dispone ampliamente de la presentación oral por lo cual se administra dosis equivalentes y fraccionadas de otros corticoides sintéticos como la prednisolona, dado que su acción dura entre 12 a 36 horas puede darse a una dosis única en la mañana de 3-5 mg/día. Su titulación depende de la respuesta clínica, evaluando estigmas de Cushing iatrogénico, sangrado, atrofia de la piel, plétora facial, intolerancia a la glucosa que indican necesidad de reducción en dosis; y por el contrario la pérdida de peso, astenia, anorexia y debilidad cuando existen dosis subterapéuticas $(7,8)$. Algunos pacientes tienen mejor respuesta con el uso de fludrocortisona de $0.05-0.2 \mathrm{mg} /$ día, pudiéndose tomar en cualquier momento del día o en días alternos cuando se usan bajas dosis (9). La suspensión del esteroide debe ser gradual con el fin de evitar reactivación de la enfermedad subyacente, una crisis adrenal y para dar más tiempo de recuperación a las glándulas suprarrenales; sin embargo, no hay suficiente evidencia de la eficacia de un régimen de supresión específico, el cual debe ser individualizado $(2,7)$.

A continuación presentamos el caso de una paciente, quien desarrolló SC e IA luego de recibir tratamiento con artrivid PLUS. La mejoría del dolor articular retrasó su consulta hasta dos años después cuando tuvo aumento excesivo de peso e hipertensión.

\section{Caso clínico}

Paciente femenina de 67 años con antecedente de dislipidemia, menopausia a los 52 años sin terapia de reemplazo hormonal y osteoartrosis de rodillas, quien estaba recibiendo tratamiento con artrivid PLUS dos tabletas diarias, un producto natural homeopático comercializado para uso en enfermedad articular. Consultó por ganancia excesiva de peso a pesar de una ingesta normal de calo-

Tabla 1. Resultados de laboratorio

\begin{tabular}{|c|c|c|}
\hline Estudio & Primera revisión & Última revisión \\
\hline Calcio total (mg/dL) & 9.6 & 9.2 \\
\hline Cloro (mEq/l) & 103.1 & 101 \\
\hline Colesterol Total (mg/dL) & 148 & \\
\hline $\mathrm{TG}(\mathrm{mg} / \mathrm{dL})$ & 161 & \\
\hline Creatinina (mg/dL) & 0.88 & 0.77 \\
\hline Depuración de creatinina (ml/min) & 67.98 & \\
\hline Fosfatasa alcalina (UI/L) & 86 & \\
\hline Glucemia basal (mg/dL) & 78 & 74 \\
\hline HBAlc (\%) & 6.46 & 5.7 \\
\hline Potasio $(\mathrm{mEq} / \mathrm{L})$ & 4.34 & 4.2 \\
\hline Albúmina (mg/dL) & 4.34 & \\
\hline Sodio $(\mathrm{mEq} / \mathrm{L})$ & 144 & 140 \\
\hline ALT (UI/L) & 27.6 & \\
\hline $\operatorname{AST}(\mathrm{UI} / \mathrm{L})$ & 23 & \\
\hline $\mathrm{Hb}(\mathrm{g} / \mathrm{dL})$ & 13.5 & 13 \\
\hline Hto $(\%)$ & 43 & 42 \\
\hline Leucocitos $\left(\mathrm{cm}^{3}\right)$ & 12580 & 9800 \\
\hline Plaquetas $\left(\mathrm{cm}^{3}\right)$ & 239000 & 245000 \\
\hline Linfocitos (\%) & 27.1 & 25 \\
\hline Parcial de orina & normal & \\
\hline Cortisol en suero (ug/dL) & 0.78 & 13.7 \\
\hline $\mathrm{TSH}(\mathrm{U} / \mathrm{l})$ & 6.94 & \\
\hline T4 libre (ng/dL) & 1.22 & \\
\hline DHEA-s & 1.73 & \\
\hline Estradiol $(\mathrm{pg} / \mathrm{mL})$ & $<5$ & \\
\hline FSH & 59.9 & \\
\hline LH & 26 & \\
\hline PTH (ng/L) & 27.8 & \\
\hline
\end{tabular}


rías en la dieta y sin cambios en su actividad física. Peso mínimo conocido de 60 kilogramos. Al examen físico se documentó un patrón central de distribución de la grasa, presión arterial de $150 / 80 \mathrm{mmHg}$, peso de $80 \mathrm{~kg}$ y talla de $160 \mathrm{~cm}$, con IMC de 31.25. Los estudios de laboratorio (Tabla 1) mostraron una función tiroidea normal, cortisol sérico en ayunas suprimido, leucocitosis sin evidencia de un foco infeccioso clínico, iones sin alteraciones y una glicemia en ayunas normal pero con hemoglobina glicada en rango de prediabetes. No fue posible realizar prueba con ACTH en ese momento, pero dado el perfil clínico y bioquímico, se sospechó una supresión crónica del eje suprarrenal por uso de artrivid que explicaba el cuadro de Cushing exógeno. Se difirió el tratamiento hasta resolver el cuadro de supresión y se suspendió el artrivid. Dos días después la paciente consultó al servicio de urgencias por fatiga, malestar general, incapacidad para realizar actividad física, nauseas, dolor abdominal y vómito. A su ingreso tuvo signos de deshidratación sin trastorno electrolítico, y ante la sospecha de una crisis adrenal se inició suplencia de prednisolona $10 \mathrm{mg}$ día vía oral con desmonte gradual diario de $2.5 \mathrm{mg}$. Los dos primeros días hubo franca mejoría de los síntomas gastrointestinales, pero con la titulación de dosis presentó recaída. Dado esto se reinició predsnisolona $10 \mathrm{mg}$ día nuevamente con un plan de desmonte lento de $2.5 \mathrm{mg}$ semanal, logrando retiro de suplencia de esteroides aproximadamente a los seis meses de la primera valoración, con mejoría significativa de sus síntomas iniciales, niveles de cortisol basal de $13.7 \mathrm{ug} / \mathrm{dL}$, control de peso y disminución de niveles de hemoglobina glicosilada hasta $5.7 \mathrm{ug} / \mathrm{dL}$. Por sus síntomas articulares recibió valoración por servicio de ortopedia con diagnostico de osteoartrosis severa, requiriendo reemplazo articular de rodillas, con mejoría significativa de sus síntomas.

\section{Discusión}

Presentamos el caso de una paciente quien desarrolló hipercortisolismo exógeno e IA secundaria al consumo de artrivid PLUS. La frecuencia de administración iatrogénica de corticoides en terapia alternativa y medicina natural no es clara, pues muchos medicamentos tienen una composición parcialmente conocida donde los esteroides pueden cumplir un papel relevante por su efecto antiinflamatorio, inmunomodulador y regulador del estado de ánimo. Existen descripciones en la literatura del análisis de medicamentos o compuestos naturales donde se evidencia cierto grado de porcentaje de esteroides en su contenido, que no había sido descrito con anterioridad, presentando eventos adversos serios como hipercortisolismo exógeno, infecciones, sepsis, crisis adrenal, diabetes o intolerancia a la glucosa $(2,4,10)$.

A la fecha no existen reportes de Cushing exógeno por artrivid, nosotros describimos el primer caso, sin omitir la variabilidad que puede presentarse respecto a la concentración del producto y susceptibilidad individual a su acción. El artrivid, es un producto natural basado en dos componentes PLUS I que incluye: taraxacum officinale $15 \%$, artrocel $15 \%$, maytenus laevis reissek $15 \%$, encima tomentosa $15 \%$, uncaria tomentosa $10 \%$, glucosamine $15 \%$, aticta $15 \%$ y PLUS II con: smila officinalis $15 \%$, traumel $15 \%$, jacaranda - caucana $15 \%$, stoclal $10 \%$, salix alb15 $\%$, condroitina $15 \%$, boswella $15 \%$. Un estudio en ratones demostró que el taraxacum officinale también conocido como dandelion (planta cultivada en Asia), tiene un efecto antidepresivo a través de la modulación del sistema neuroendocrino disminuyendo los niveles séricos de cortisol y CRH, elevados en respuesta al estrés (11). No hay descripción de la acción de los elementos restantes sobre el eje, ni tampoco del contenido específico de esteroides sintéticos. En el caso que describimos los síntomas presentados en la paciente y adicionalmente la respuesta a su suspensión nos hicieron sospechar un SC e IA.

El SC exógeno está asociado con alta tasa de morbilidad y mortalidad, la historia clínica proporciona aspectos clave pues la relación temporal del consumo con los síntomas y signos y su suspensión, hacen sospechar esta causa inusual. Idealmente complementar el estudio de cortisol sérico con medición de cortisol urinario, valores de ACTH suprimidos, no respuesta al estímulo de ACTH y la evidencia de atrofia adrenal en imagen, aumentan la sensibilidad del diagnóstico, pero en este caso no realizamos una evaluación dinámica del eje pues los síntomas graves de la paciente impidieron retardar el tratamiento $(2,4)$. El análisis con espectrometría de masas y cromatografía líquida es una técnica que permite la detección simultánea y cuantitativa de esteroides sintéticos en plasma, orina y tabletas, desafortunadamente, no contamos con la disponibilidad en nuestro medio para poder determinar la concentración exacta de esteroides en Artrivid PLUS; nosotros acertamos en el diagnóstico de una fuente exógena de esteroides a través de una prueba terapéutica (10).

El artrivid PLUS no cuenta con registro sanitario expedido por el Instituto Nacional de Vigilancia de Medicamentos y Alimentos (INVIMA) para su uso terapéutico, en el 2015 comunicaron que su composición legítima es desconocida y se instruyó sobre el reporte de efectos adversos relacionados con su consumo (12). Consideramos que su uso puede producir SC y supresión adrenal prolongada, con desconocimiento de las reacciones adversas adicionales que puede desencadenar, por lo que se debe continuar implementando medidas preventivas en la regulación de su comercialización.

Ninguno

\section{Conflictos de interés}

\section{Conclusión}

El síndrome de Cushing exógeno e insuficiencia adrenal relacionados a la inclusión de corticoides en medicamentos y productos naturales de uso común, es una condición que 
debe ser reconocida por el clínico, pues la sospecha y suspensión temprana del medicamento permitirán impactar en la morbilidad y mortalidad, así como una consecuente alerta para frenar la divulgación y aceptación de su uso.

\section{Referencias}

1. Nieman L. Cushing's syndrome: update on signs, symptoms and biochemical screening. Eur J Endocrinol. 2015; 173(4): 33-8.

2. Charmandari E, Nicolaides N, Chrousos G. Adrenal insufficiency. Lancet. 2014; 383(9935): 2152-67.

3. Dinsen S, Baslund B, Klose M, Rasmussen A, Friis-Hansen L, Hilsted L, et al. Why glucocorticoid withdrawal may sometimes be as dangerous as the treatment itself. Eur J Intern Med. 2013; 24(8): 714-20

4. Harvey P. Adrenocortical endocrine disruption. J Steroid Biochem Mol Biol. 2016; 155(Pt B): 199-206.

5. Joseph R, Hunter A, Ray D, Dixon W. Systemic glucocorticoid therapy and adrenal insufficiency in adults: A systematic review. Semin Arthritis Rheum. 2016; 9.

6. Raff H, Sharma S, Nieman L. Physiological basis for the etiology, diagnosis, and treatment of adrenal disorders: Cushing's syndrome, adrenal insufficiency,and congenital adrenal hyperplasia. Compr Physiol. 2014; 4(2): 739-69.

7. Bornstein S, Allolio B, Arlt W, Barthel A, Don-Wauchope A, Hammer G, et al Diagnosis and Treatment of Primary Adrenal Insufficiency: An Endocrine Society Clinical Practice Guideline. J Clin Endocrinol Metab. 2016; 101(2): 364-89

8. Øksnes M, Ross R, Løvås K. Optimal glucocorticoid replacement in adrenal insufficiency. Best Pract Res Clin Endocrinol Metab. 2015; 29(1): 3-15.

9. Quinkler M, Oelkers W, Remde H, Allolio B. Mineralocorticoid substitution and monitoring in primary adrenal insufficiency. Best Pract Res Clin Endocrinol Metab. 2015; 29(1): 17-24.

10. Azizi F, Jahed A, Hedayati M, Lankarani M, Bejestani H, Esfahanian F, et al. Outbreak of exogenous Cushing's syndrome due to unlicensed medications. Clin Endocrinol (Oxf). 2008; 69(6): 921-5

11. Li Y, Shen J, Li Y, Huang Q. Antidepressant effects of the water extract from Taraxacum officinale leaves and roots in mice. Pharm Biol. 2014; 52(8): 1028-32.

12. Información de seguridad sobre el producto "Artrivid Plus" promocionado en diferentes medios de comunicación del país. Alerta sanitariadirección de medicamentos y productos biológicos. 2015; [3 páginas].Disponible en: https://www. invima.gov.co 